\title{
Simulation Analysis of Extended Kalman Filter Applied for Estimating Position and Speed of a Brushless DC Motor
}

Research Article

\author{
Maciej Chojowski
}

AGH University of Science and Technology, Al. Adama Mickiewicza 30, 30-059

Kraków, Poland

Received March 07, 2018; Accepted May 31, 2018

Abstract: The purpose of this paper was to present a method for the estimation of the rotor speed and position of brushless DC (BLDC) motor. The BLDC motor state equations were developed, and the model was discretised. Extended Kalman filter has been designed to observe specific states from the state vector, needed for the sensorless control (rotor position) and to determine the speed, which may be useful to use as a feedback for the controller. A test was carried out to determine the noise covariance matrices in a simulation manner.

Keywords: extended Kalman filter • brushless DC motor • covariance matrix • sensorless control for BLDC

\section{Introduction}

Brushless DC (BLDC) motors are increasingly used in recent years. Simple methods of developing the control system (high similarity in the development of the control system as in the motor with mechanical commutator) have resulted in driving with BLDC motors much more advantageous than that with the classic DC motors. The main advantages of the BLDC motors are high efficiency, high durability and elimination of the mechanical commutator with brushes. The disadvantage of the BLDC motors is the pulsation of the generated electrical torque. The increase in interest in the BLDC motor drive results in the search for new motor control methods and the estimation of the rotor position in order to eliminate Hall sensors. One of the methods of position estimation is the application of a Kalman filter, which plays a significant role in industry and is still the subject of scientific research (Aishwarya and Jayanand, 2016; Lenine et al., 2007; Terzic and Jadric, 2001; Zabalawi and Nasiri, 2007). The utilisation of estimated speed and angle can be used as a feedback for BLDC motor control system, which has already been presented in Lenine et al. (2007). There are still ongoing studies to improve the control using the optimal estimator, which was presented in Aishwarya and Jayanand (2016), where the PFC buck-boost converter and three-phase bridge were developed. In recent years, research related to observers are heading to identify fault in BLDC motors, described in Eissa et al. (2015) and El Mekki and Ben Saad (2016). We can also find H infinity approach for realisation of robust sensorless drive (Vinida and Chacko, 2016). The aim of this paper was dedicated to the application of Kalman filter in the BLDC motor. A precise investigation will be performed to achieve determination of best covariance matrix. 


\section{BLDC motor model}

Three-phase permanent magnet BLDC motor can be modelled as a series connection of resistance, induction and voltage source. Back EMF represents the effect of the rotor on the stator side. The mathematical model can be represented as a differential equation with constant parameter values (Aishwarya and Jayanand, 2016; Jethwani et al., 2016; Lenine et al., 2007; Terzic and Jadric, 2001; Zabalawi and Nasiri, 2007). The voltage matrix equation for a three-phase BLDC motor is as follows (assuming symmetries in every phase):

$$
\left[\begin{array}{c}
U_{a} \\
U_{b} \\
U_{c}
\end{array}\right]=\left[\begin{array}{ccc}
R_{S} & 0 & 0 \\
0 & R_{S} & 0 \\
0 & 0 & R_{S}
\end{array}\right]\left[\begin{array}{c}
i_{a} \\
i_{b} \\
i_{c}
\end{array}\right]+\left[\begin{array}{ccc}
L & 0 & 0 \\
0 & L & 0 \\
0 & 0 & L
\end{array}\right] \frac{d}{d t}\left[\begin{array}{c}
i_{a} \\
i_{b} \\
i_{c}
\end{array}\right]+\left[\begin{array}{c}
e_{a} \\
e_{b} \\
e_{c}
\end{array}\right]
$$

The stator currents of the motor satisfy the following equation:

$$
i_{a}+i_{c}+i_{b}=0
$$

where $R_{S}$ is the phase resistance, $L$ the one-phase inductance $\left(L_{S}-M\right), L_{S}$ the stator self-inductance and $M$ the mutual inductance.

The electromotive force has a trapezoidal shape, and its values can be recorded on the basis of Table 1 . Function $f_{b}(\varphi)$ and $f_{c}(\varphi)$ are shifted by $\frac{2}{3} \pi$ and $\frac{4}{3} \pi$, respectively.

Table 1. The values of the electromotive force as a function of the elctrical angle

\begin{tabular}{cc}
$\varphi$ & $f_{a}(\varphi)$ \\
$0<\varphi<\frac{\pi}{3}$ & 1 \\
$\frac{\pi}{3}<\varphi<\frac{2 \pi}{3}$ & $6\left(\frac{\pi}{3}-\varphi\right) / \pi$ \\
$\frac{2 \pi}{3}<\varphi<\frac{4 \pi}{3}$ & -1 \\
$\frac{4 \pi}{3}<\varphi<\frac{5 \pi}{3}$ & $6\left(\varphi-3 \frac{\pi}{2}\right) / \pi$ \\
$\frac{5 \pi}{3}<\varphi<2 \pi$ & 1 \\
\hline
\end{tabular}

The maximum value of the electromotive force is given by the following formula:

$$
E_{\text {peak }}=k_{w} \omega_{m}
$$

where $\omega_{m}$ is the mechanical speed and $k_{w}$ the back EMF constant.

The values of electromotive force as a function of the electric angle from Table 1 and equation (3) can be written as:

$$
\begin{aligned}
& e_{a}=E_{\text {peak }} f_{a}(\varphi) \\
& e_{b}=E_{\text {peak }} f_{b}(\varphi)
\end{aligned}
$$




$$
e_{c}=E_{\text {peak }} f_{c}(\varphi)
$$

The value of the electrical torque generated by the BLDC motor can be represented by the following equation (Aishwarya and Jayanand, 2016; Lenine et al., 2007; Terzic and Jadric, 2001; Zabalawi and Nasiri, 2007):

$$
T_{e}=\frac{e_{a} i_{a}+e_{b} i_{b}+e_{c} i_{c}}{\omega_{m}}
$$

By substituting equations (4)-(6) to (7), the equation for torque is as follows:

$$
T_{e}=k_{w}\left(f_{a}(\varphi) i_{a}+f_{b}(\varphi) i_{b}+f_{c}(\varphi) i_{c}\right)
$$

The mechanical equation of the motor model is given by Aishwarya and Jayanand (2016) as follows:

$$
J \frac{d \omega_{m}}{d t}=T_{e}-T_{l}
$$

Numerous publications describe the mechanical equation together with the coefficient of friction, which is a simplification, because the model becomes a two-inertia system by replacing integration.

The value of th electrical angle is calculated based on the following equation (Eissa et al., 2015; Lenine et al., 2007; Terzic and Jadric, 2001; Zabalawi and Nasiri, 2007):

$$
\frac{d \varphi}{d t}=n_{p} \omega_{m}
$$

where $n_{p}$ are pole pairs.

The equations were implemented in the MATLAB Simulink programme using the SimPowerSystems library (Matlab Simulink, 2018). A model of a resistance, inductance and electromotive force (RLE) system with a controlled voltage source was a model of a resistance, inductance and electromotive force (RLE) system with a controlled voltage source was developed (Jethwani et al., 2016). The trapezoidal voltage shape was obtained by writing $f(\varphi)$ values in the Lookup table (based on Table 1). Fig 1. shows brushless dc motor model.

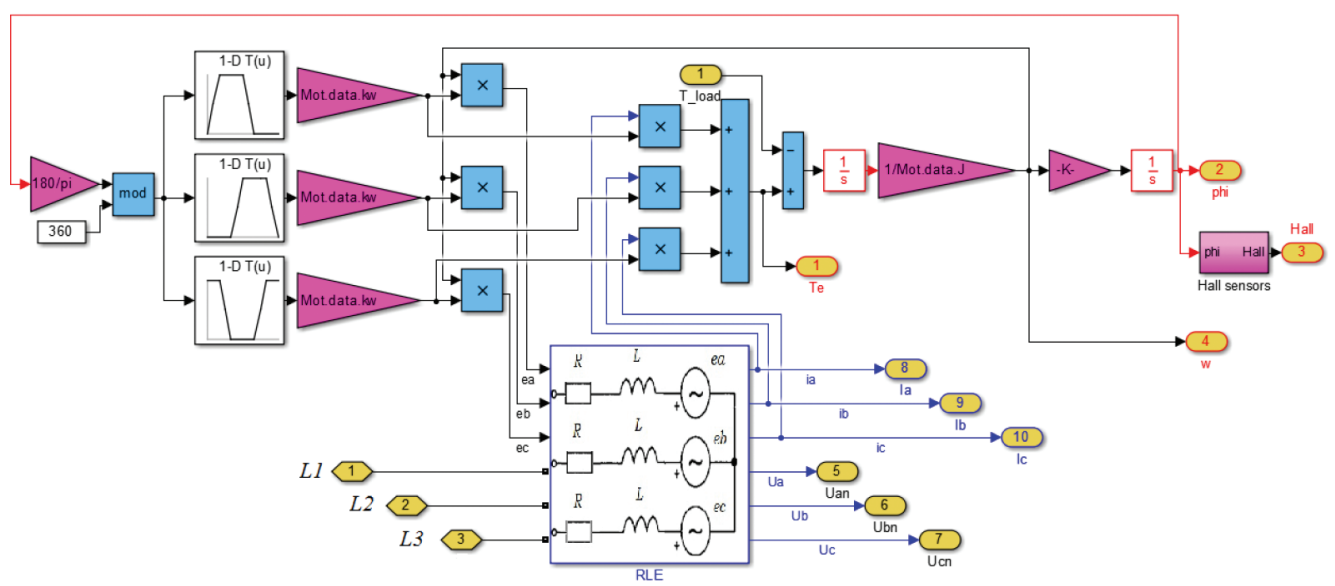

Fig. 1. $B L D C$ motor model - Simulink implementation. Model inputs $(L 1, L 2, L 3)$ are from power electronic $D C / A C$ converter

Based on previous equations, a full BLDC motor model can be written as a state equation (Aishwarya and Jayanand, 2016; Terzic and Jadric, 2001; Zabalawi and Nasiri, 2007): 


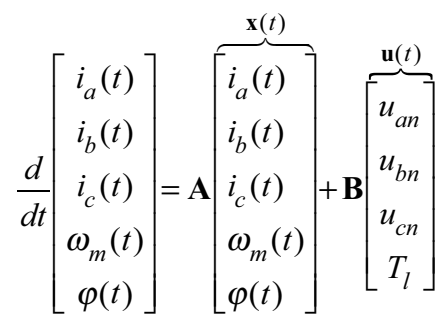

$$
\begin{aligned}
& \overbrace{\left[\begin{array}{c}
i_{a}(t) \\
i_{b}(t) \\
i_{c}(t)
\end{array}\right]}^{\mathbf{y}(t)}=\mathbf{C}\left[\begin{array}{c}
i_{a}(t) \\
i_{b}(t) \\
i_{c}(t) \\
\omega_{m}(t) \\
\varphi(t)
\end{array}\right]+\mathbf{D}\left[\begin{array}{c}
u_{a n} \\
u_{b n} \\
u_{c n} \\
T_{l}
\end{array}\right]
\end{aligned}
$$

where the input and output matrices are given as follows:

$$
\begin{aligned}
& \mathbf{A}=\left[\begin{array}{ccccc}
\frac{-R_{s}}{L} & 0 & 0 & \frac{-k_{w}}{L} f_{a}(\varphi) & 0 \\
0 & \frac{-R_{s}}{L} & 0 & \frac{-k_{w}}{L} f_{b}(\varphi) & 0 \\
0 & 0 & \frac{-R_{s}}{L} & \frac{-k_{w}}{L} f_{c}(\varphi) & 0 \\
\frac{k_{w}}{J} f_{a}(\varphi) & \frac{k_{w}}{J} f_{b}(\varphi) & \frac{k_{w}}{J} f_{c}(\varphi) & 0 & 0 \\
0 & 0 & 0 & n_{p} & 0
\end{array}\right] \\
& \mathbf{B}=\left[\begin{array}{cccc}
\frac{1}{L} & 0 & 0 & 0 \\
0 & \frac{1}{L} & 0 & 0 \\
0 & 0 & \frac{1}{L} & 0 \\
0 & 0 & 0 & -\frac{1}{J} \\
0 & 0 & 0 & 0
\end{array}\right], \mathbf{C}=\left[\begin{array}{ccccc}
1 & 0 & 0 & 0 & 0 \\
0 & 1 & 0 & 0 & 0 \\
0 & 0 & 1 & 0 & 0
\end{array}\right]
\end{aligned}
$$

Feedforward matrix (i.e. D) is full of zeroes in the BLDC motor model. If the continuous system is sampled with interval, which is at least ten times shorter than the motor stator time constant, then discrete state space system can be obtained by Euler's method (Terzic and Jadric, 2001). Equations (11) and (12) after discretisation over time, sampling $T_{S}$, takes the following form:

$$
\begin{aligned}
& \mathbf{x}(k+1)=\left(\mathbf{I}+T_{s} \mathbf{A}\right) \mathbf{x}(k)+T_{s} \mathbf{B u}(k)=\mathbf{A}_{D} \mathbf{x}(k)+\mathbf{B}_{D} \mathbf{u}(k) \\
& \mathbf{y}(k)=\mathbf{C x}(k)
\end{aligned}
$$


Discrete model equations (16) and (17) are used to develop a Kalman filter to estimate the states of the motor. The BLDC motor parameters used in further simulations are included in Table 2.

Table 2. Motor specification

\begin{tabular}{cccc}
\hline Motor parameter & Symbol & Value & Unit \\
\hline \hline Resistance per phase & $R_{S}$ & 4.95 & $\Omega$ \\
Inductance $\left(L_{S}-M\right)$ & $L$ & 2.1 & $\mathrm{mH}$ \\
Back EMF constant & $k_{w}$ & 55.21 & $\mathrm{mVs}$ \\
Moment of inertia & $J$ & 16.17 & $\mathrm{mkgm}^{2}$ \\
No of pole pair & $n_{p}$ & 4 & - \\
\hline
\end{tabular}

The simulation was run for half a second. The motor was driven at no load till $0.1 \mathrm{sec}$ and then a load torque of $0.38 \mathrm{Nm}$ was introduced. Figure 2 presents mechanical speed and torque as function of time.
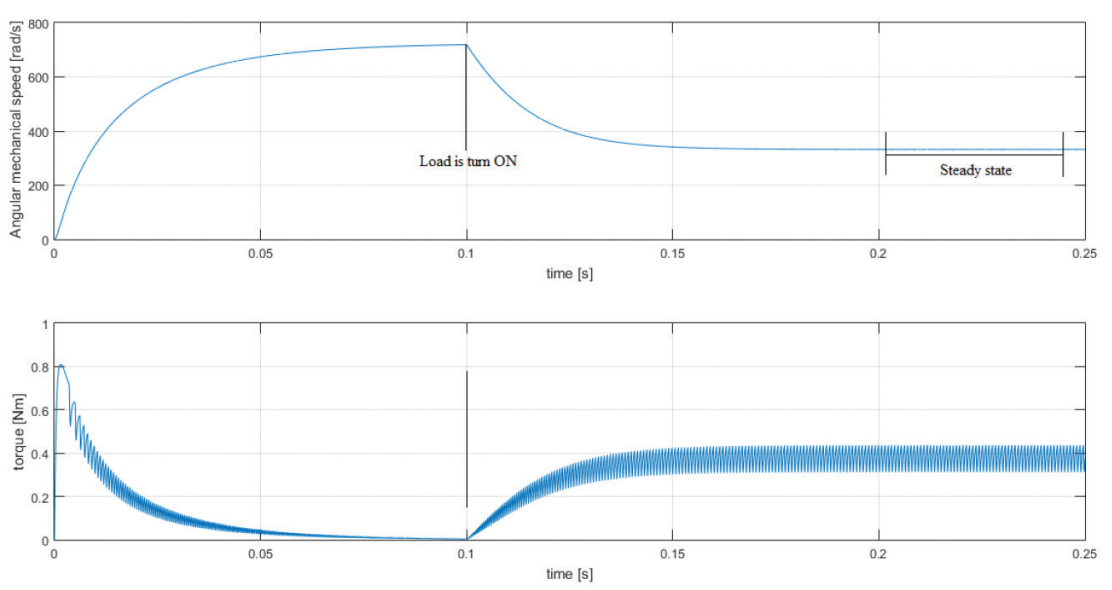

Fig. 2. Mechanical speed and driving torque as function of time

It is important to notice that the load torque value will be treated as the known input during simulation, but in a real drive application, a load torque value is seldom known. It is possible to measure the value of the torque, but the sensor cost is usually high, which causes a high price for the drive. A possible solution is to treat our load torque not as an input but as disturbance to be observed by the observer.

\section{Extended Kalman filter (EKF)}

According to the presented solution by Rudolf Emil Kalman, to develop a filter, a mathematical model of the system based on differential equations should be created (Auger et al., 2013; Simon, 2006). The Kalman filter estimate the state vector $\hat{\mathbf{x}}(t)$ on the basis of the model together with the measurement of the input vector $\mathbf{u}(t)$ and the output vector $\mathbf{y}(t)$.

According to the assumption, the motor model is given by the following equations of state vector:

$$
\begin{aligned}
& \frac{d}{d t} \mathbf{x}(t)=\mathbf{A} \mathbf{x}(t)+\mathbf{B u}(t)+\mathbf{w}(t) \\
& \mathbf{y}(t)=\mathbf{C x}(t)+\mathbf{v}(t),
\end{aligned}
$$

where $\mathbf{w}(t)$ and $\mathbf{v}(t)$ are white noise with zero mean, uncorrelated with covariance matrices ( $\mathbf{Q}$ and $\mathbf{R}$, respectively) (Simon, 2006). The process covariance matrix (i.e. Q), the measurement covariance matrix (i.e. R) and the initial values of the estimation error covariance matrix (i.e. P) together with the initial values of the state vector should be selected. There are several methods for selecting the matrix value. In the case of correlated noise, the entire matrix 
will have elements (Simon, 2006). The values in the matrix will depend on measurement noise covariance. If the signals are correlated with known variance of the noise, then matrix $\mathbf{R}$ is defined as:

$$
\mathbf{R}=\left(\begin{array}{ccc}
\sigma_{n}^{2} & \cdots & \sigma_{n} \sigma_{m} \\
\vdots & \ddots & \vdots \\
\sigma_{n} \sigma_{m} & \cdots & \sigma_{m}^{2}
\end{array}\right)
$$

where $\sigma_{m}$ and $\sigma_{n}$ denote variance of the measurement noise ( $m$ and $n$ are positive integer numbers determining size of the matrix) and $\sigma_{n} \sigma_{m}$ the covariance of the signals.

The $\mathbf{R}$ matrix can be determined on the basis of measurement noise, where the values on the diagonal correspond to the variance of the measurement noise. The $\mathbf{Q}$ matrix is responsible for the process noise in the model and should be selected after determining the matrix $\mathbf{R}$ (Dhaouadi et al., 1991).

Next, covariance matrices are selected in this case, uncorrelated measurement. In this case, $\mathbf{R}$ matrix is much simpler (Aishwarya and Jayanand, 2016; Dhaouadi et al., 1991; Terzic and Jadric, 2001):

$$
\mathbf{R}=\left(\begin{array}{ccc}
\sigma_{n}^{2} & \cdots & 0 \\
\vdots & \ddots & \vdots \\
0 & \cdots & \sigma_{m}^{2}
\end{array}\right)=\operatorname{diag}\left(\sigma_{n}^{2}, \ldots, \sigma_{m}^{2}\right)
$$

If the model is not given in relative units and the state vectors consist of different sizes (e.g., current, speed, position), it is necessary to transfer to relative units in order for the Kalman Filter to work properly and increase value that we want to optimise:

$$
\mathbf{Q}=\operatorname{diag}\left(\frac{1}{X_{1-\max }}, \frac{1}{X_{2-\max }}, \ldots, \frac{1}{X_{n-\max }}\right)
$$

The process covariance matrix can be also selected based on the following formula (Ryba et al., 2014):

$$
\mathbf{Q}=\mathbf{B} \cdot \mathbf{B}^{\prime}
$$

where: B - input matrix

The measurement covariance matrix can be selected based on the variance of the measurement noise. The value of covariance matrix $\mathbf{R}$ can be selected based on the measured data or based on the parameters of the sensor. Detailed calculation of the standard deviation for the mechanical speed measurement by incremental encoder can be calculated as follows (Šlapák et al., 2016):

$$
\sigma_{\omega}=\frac{1}{2} \cdot\left(\frac{2 \pi}{4 \cdot N \cdot T_{E N}}\right)
$$

where $T_{E N}$ is the sample time of the encoder and $N$ the number of pulses per revolution.

The Kalman filter was invented to estimate the parameters of the linear model described by the state equations. The system representing the BLDC motor was non-linear, so the EKF was used, which was developed to observe the parameters of the non-linear model. Substituting the first two parts of the Taylor series to the state space model (Aishwarya and Jayanand, 2016; Dhaouadi et al., 1991; Lenine et al., 2007; Simon, 2006; Terzic and Jadric, 2001):

$$
\frac{d}{d t} \hat{\mathbf{x}}(t)=\overbrace{\nabla \cdot[\mathbf{A} \hat{\mathbf{x}}(t)+\mathbf{B u}(t)]}^{\mathbf{f}(\hat{\mathbf{x}}, \mathbf{u})}]_{\mathbf{x}=\mathbf{x}_{\mathbf{0}}, \mathbf{u}=\mathbf{u}_{\mathbf{0}}}=\mathbf{f}(\hat{\mathbf{x}}, \mathbf{u})_{\mathbf{x}=\mathbf{x}_{\mathbf{0}}, \mathbf{u}=\mathbf{u}_{\mathbf{0}}}
$$


where $\nabla$ is the Jacobian matrix and $\mathbf{x}_{\mathbf{0}}, \mathbf{u}_{\mathbf{0}}$ are state and control vector values in specific point. The derivative of nonlinear state space equation is as follows:

$$
\mathbf{f}(\hat{\mathbf{x}}, \mathbf{u})=\left[\begin{array}{ccccc}
\frac{-R_{s}}{L} & 0 & 0 & \frac{-k_{w}}{L} f_{a}(\varphi) & \frac{-k_{w}}{L} \frac{d f_{a}(\varphi)}{d \varphi} \\
0 & \frac{-R_{s}}{L} & 0 & \frac{-k_{w}}{L} f_{b}(\varphi) & \frac{-k_{w}}{L} \frac{d f_{b}(\varphi)}{d \varphi} \\
0 & 0 & \frac{-R_{s}}{L} & \frac{-k_{w}}{L} f_{c}(\varphi) & \frac{-k_{w}}{L} \frac{d f_{c}(\varphi)}{d \varphi} \\
\frac{\omega k_{w}}{J} f_{a}(\varphi) & \frac{\omega k_{w}}{J} f_{b}(\varphi) & \frac{\omega k_{w}}{J} f_{c}(\varphi) & 0 & \Sigma \\
0 & 0 & 0 & n_{p} & 0
\end{array}\right]
$$

The value of $\sum=\frac{-\omega k_{w}}{J}\left(\frac{d f_{a}(\varphi)}{d \varphi}+\frac{d f_{b}(\varphi)}{d \varphi}+\frac{d f_{c}(\varphi)}{d \varphi}\right)$. The derivative of the angle function has been calculated based on Table 1. One of the values of the function is given in Table 3.

Table 3. Derivative of $f_{a}(\varphi)$

\begin{tabular}{cc}
$\varphi$ & $d f_{a}(\varphi) / d \varphi$ \\
$0<\varphi<\frac{\pi}{3}$ & 0 \\
$\frac{\pi}{3}<\varphi<\frac{2 \pi}{3}$ & $-6 / \pi$ \\
$\frac{2 \pi}{3}<\varphi<\frac{4 \pi}{3}$ & 0 \\
$\frac{4 \pi}{3}<\varphi<\frac{5 \pi}{3}$ & \\
$\frac{5 \pi}{3}<\varphi<2 \pi$ & $6 / \pi$ \\
\hline
\end{tabular}

The initial values of the matrix $\mathbf{P}$ with the initial values of the state vector were selected to zero. The algorithm of the discrete EKF has been divided into appropriate steps (Simon, 2006):

\section{Step 1: Prediction of the state vector}

The state vector at sampling time $k+1$ was predicted from the input vector $\mathbf{U}$ and the state vector $\mathbf{X}$ at previous sampling time $k$ by using state space BLDC motor model:

$$
\begin{aligned}
& \mathbf{X}_{k+1}^{-}=\mathbf{F}_{k} \hat{\mathbf{X}}_{k}^{+}+\mathbf{B}_{k} \mathbf{U}_{K} \\
& \mathbf{P}_{k+1}^{-}=\mathbf{F}_{k} \mathbf{P}_{k}^{+} \mathbf{F}_{k}^{T}+\mathbf{Q} .
\end{aligned}
$$

In equations (27) and (28), both $\mathbf{X}_{k}^{-}$and $\mathbf{X}_{k}^{+}$are estimates of the same state vector $\mathbf{X}_{k}$ in the sampling time $k, \mathbf{X}_{k}^{-}$is estimation before the measurement $\mathbf{Y}_{k}$ is taken (a priori estimate) and $\mathbf{X}_{k}^{+}$is estimation after the measurement is taken (a posteriori estimate). $\mathbf{F}_{k}$ is discrete gradient matrix $\mathbf{f}(\hat{\mathbf{x}}, \mathbf{u})$, and $\mathbf{B}_{k}$ is discrete input matrix.

\section{Step 2: State and covariance correction based on measurements}

The measurement update equations, which try to make correction to the prediction state vector based on measurement signals at time $k+1$. The equations for Kalman filter become: 


$$
\mathbf{K}_{k+1}=\mathbf{P}_{k+1}^{-} \mathbf{C}^{T}\left(\mathbf{C} \mathbf{P}_{k+1}^{-} \mathbf{C}^{T}+\mathbf{R}\right)^{-1}
$$

The state vector update estimation after measurement at time $k+1$ :

$$
\mathbf{X}_{k+1}^{+}=\mathbf{X}_{k+1}^{-}+\mathbf{K}_{k+1}\left(\mathbf{Y}_{k+1}-\mathbf{C X}_{k+1}^{-}\right)
$$

The covariance matrix after measurement was processed at time $k+1$ :

$$
\mathbf{P}_{k+1}^{+}=\left(I-\mathbf{K}_{k+1} \mathbf{C}\right) \mathbf{P}_{k+1}^{-}
$$

The equation was used to implement the filter in the MATLAB Simulink program. EKF with the BLDC motor and inverter circuit are shown in Fig. 3.

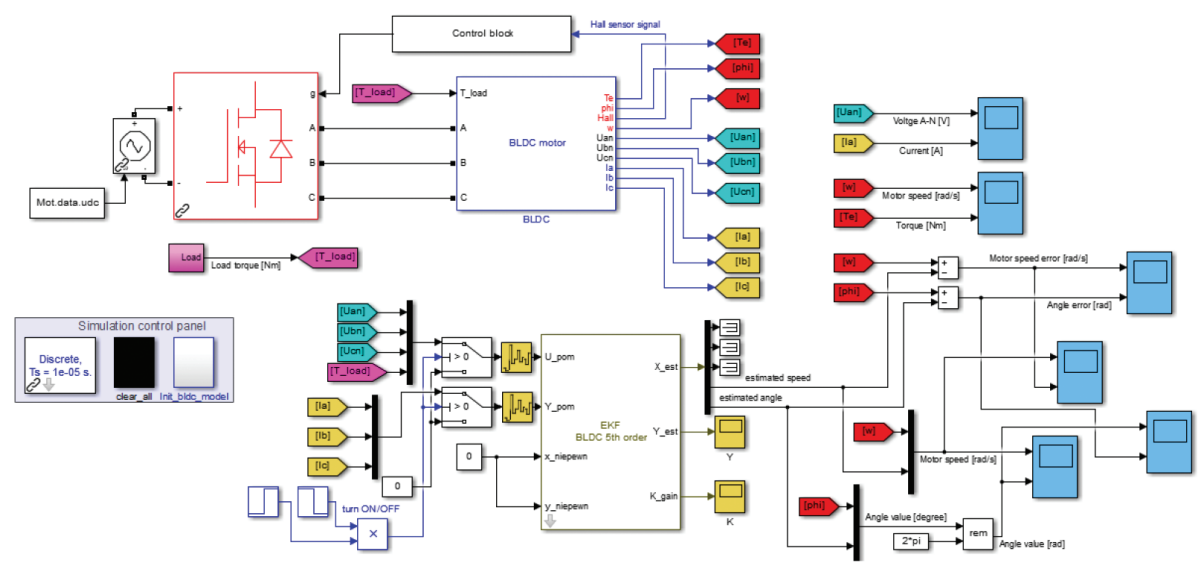

Fig. 3. Permanent-magnet BLDC motor with EKF - Simulink implementation

The BLDC motor model with the Kalman filter, which was estimated in parallel, was used to carry out simulation experiments (Fig. 2). The inverter is powered from a constant voltage source. The shape of $\mathbf{Q}$ is $5 \times 5$, and $\mathbf{R}$ is a $3 \times 3$ matrix for selected BLDC motor model.

\section{Simulation test of covariance matrix}

The Kalman filter has covariance matrices that can be used to achieve better estimation results and to improve the dynamics of the estimator. Numerous publications omit the topic of covariance matrix selection and most often choose an identity matrix. The purpose of this paper was to present methods for selecting the covariance matrices. The values of the covariance matrices were chosen in two different ways based on the previous considerations. In both cases, the measurement noise was added to measurements:

- Method 1. Simulation was carried out in which the values of the covariance matrices ( $\mathbf{Q}$ and $\mathbf{R})$ were changed. The purpose of the simulation was to find such values of the covariance matrix to get the best estimate of the angle in the steady state. The result of the test was 3D graphs, which present multiplier values of covariance matrices and error value $\left(y-y_{\text {est }}\right)$ in the steady state for loaded motor.

- Method 2. The process covariance matrix was chosen based on the formula (23), and the measurement covariance matrix was set to identity matrix.

A simulation test was carried out to determine the matrix value - method 1. During the test, the values of the $\mathbf{Q}$ and $\mathbf{R}$ matrices were changed by $q_{m}$ and $r_{m}$ covariance matrix multipliers (33) and (34). The multiplier value has been changed from $1 \cdot 10^{-2}$ to $5 \cdot 10^{-1}$ based on measurement variance. Uncorrelated measurement noise was assumed, and measurement noise power $N_{p}$ was calculated by: 


$$
N_{p}=T_{s} \cdot \sigma^{2}
$$

where $\sigma^{2}$ is the variance of the noise.

White Gaussian noise power was calculated and set to $1 \cdot 10^{-6}$. Covariance matrix with multiplier is as follows:

$$
\begin{aligned}
& \mathbf{R}=r_{m} \cdot\left[\begin{array}{lll}
1 & 0 & 0 \\
0 & 1 & 0 \\
0 & 0 & 1
\end{array}\right], \\
& \mathbf{Q}=q_{m} \cdot \operatorname{diag}\left(\frac{1}{X_{1-\max }}, \frac{1}{X_{2-\max }}, \frac{1}{X_{3-\max }}, \frac{1}{X_{4-\max }}, \frac{1}{X_{5-\max }}\right),
\end{aligned}
$$

where values are set to $X_{1-\max }=X_{2-\max }=X_{3-\max }=\frac{1}{10}$ and $X_{4-\max }=100 ; X_{5-\max }=\frac{1}{10000}$.

The estimated speed and angle were recorded to calculate the steady-state error (several recent samples from the measurement of angular speed error and rotation angle were averaged). A greater error in the steady-state condition than the given values will not be interesting to use in the control system or to make sensorless drive.

Increasing the process noise covariance matrix without increasing the measurement noise covariance matrix will result in a large estimation error for angle estimation. Increasing the value on the diagonal of the $\mathbf{R}$ matrix will reduce the estimation error, which is confirmed in Fig. 4. Figure 5 presents average mechanical speed error in steady state.

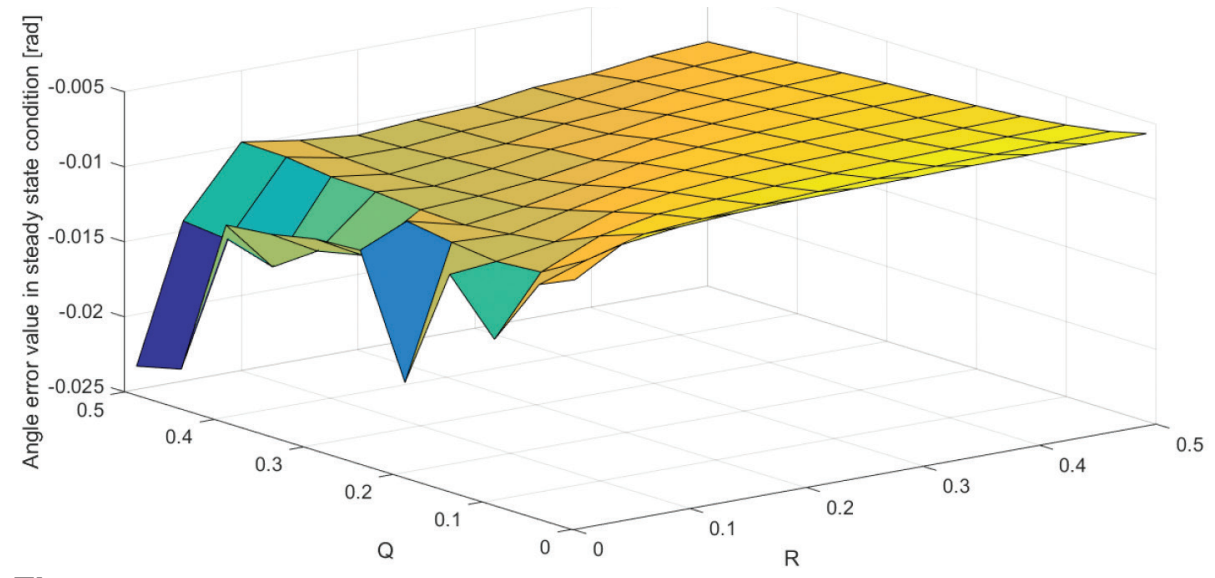

Fig. 4. The $3 D$ graph shows the average error in the steady state for the angle value [rad]

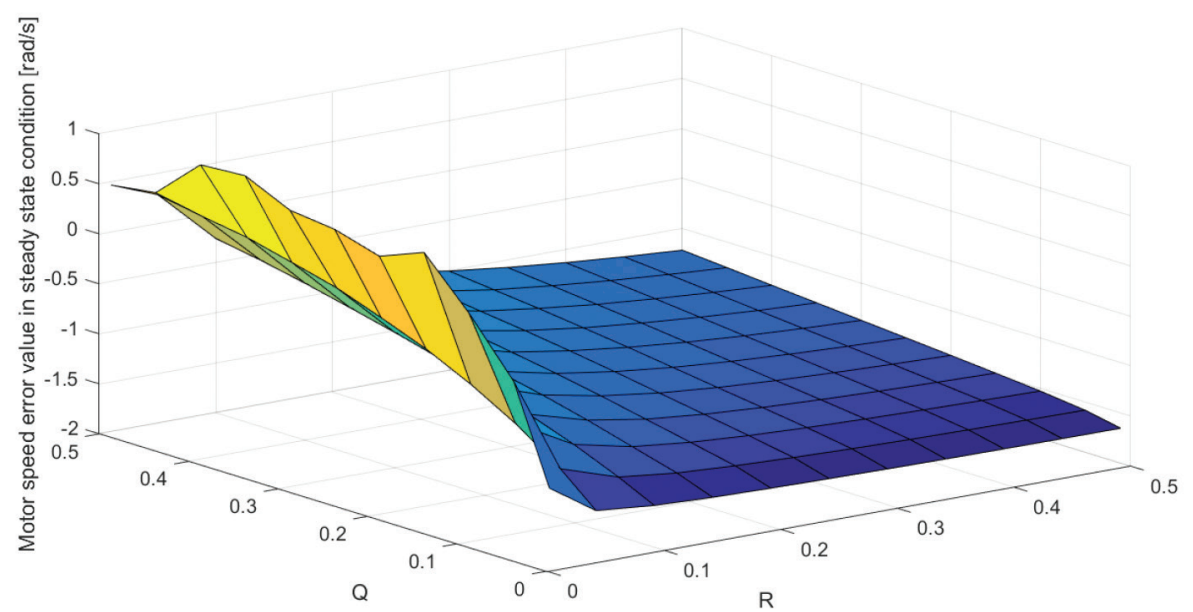

Fig. 5. The $3 \mathrm{D}$ graph shows the average error in the steady state for the speed value $[\mathrm{rad} / \mathrm{s}]$ 
A significant value in the process covariance matrix, corresponding to the speed state variable $\left(X_{4-\max }\right)$, causes an increase in the value of the $\mathbf{R}$, which does not reduce the error in the steady state (small impact). The speed is more dependent on the model than the measurements. Based on simulation results, matrix values were selected to achieve small error value in angle estimation $\left(r_{m}=0.5, q_{m}=0.01\right)$.

It should be noticed that if measurement covariance matrix is calculated, then only the value of $\mathbf{Q}$ remains unknown. Using process covariance matrix deviation, we can carry out similar simulation to obtain the desired steady-state error.

The estimated speed and angle for both methods with the error signal $\left(y-y_{\text {est }}\right)$ are presented in Figs. 6 and 7.

The simulation allows to collect a database of parallel test results necessary for assessing both method and quantifying the differences between the two methods. The maximum error $\left(\max \left|y-y_{\text {est }}\right|\right)$ for the first method in the steady state is equal to $6.26 \mathrm{rad} / \mathrm{s}$. The second method of selection covariance matrices gives a more precise result equal to $6.20 \mathrm{rad} / \mathrm{s}$ but variance (3.00) is higher in comparison with the first method (2.87).
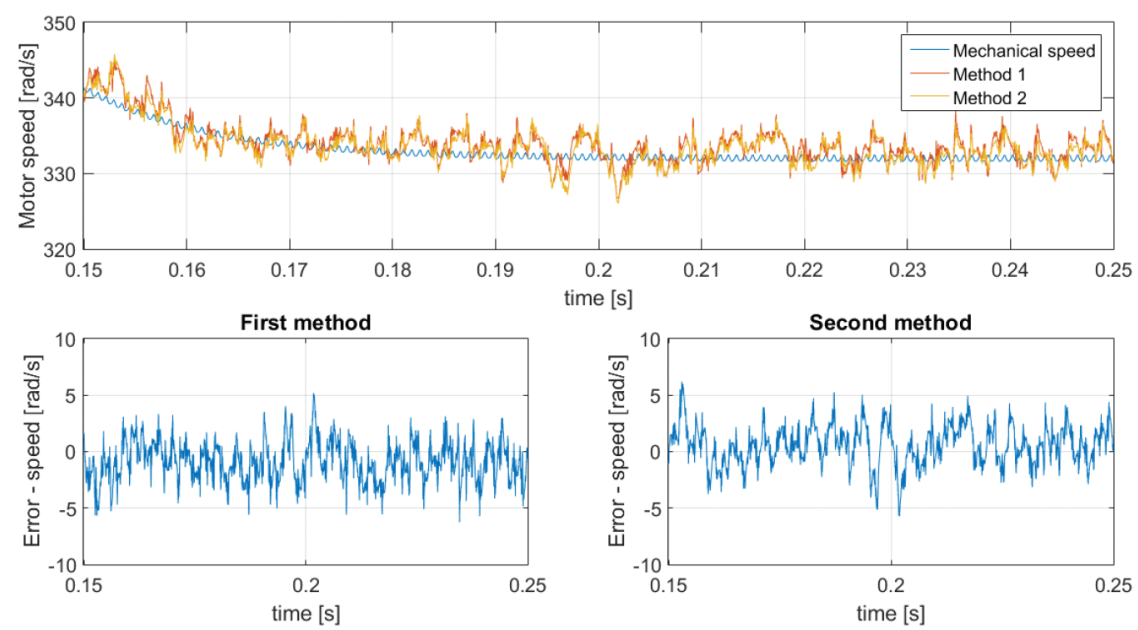

Fig. 6. Angular speed comparison and steady-state error for both methods $[\mathrm{rad} / \mathrm{s}]$
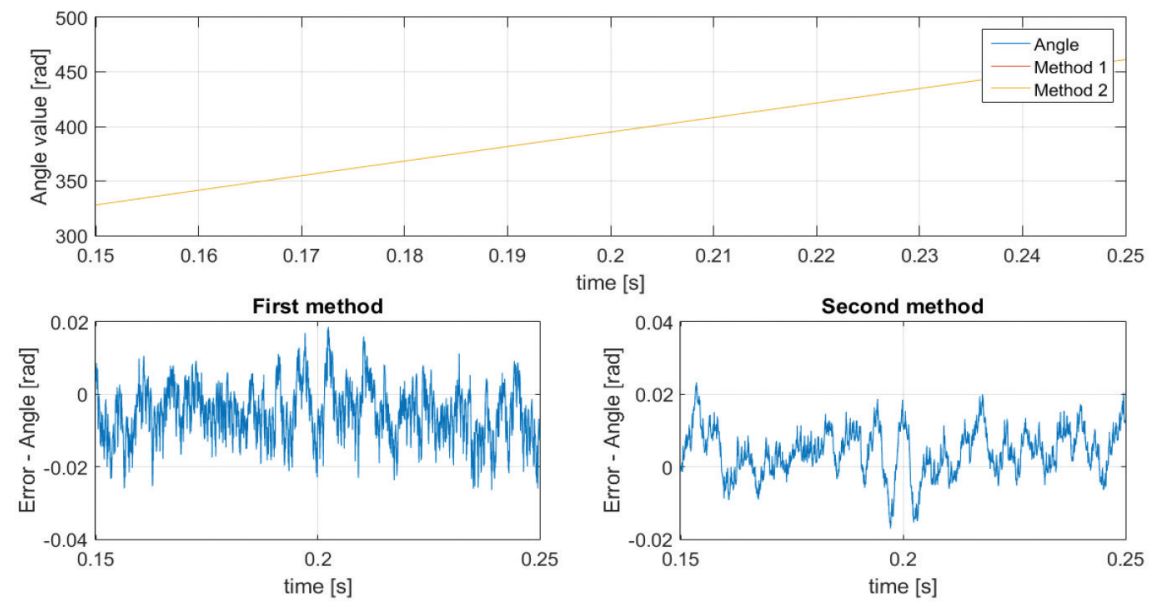

Fig. 7. Angle comparison and error value for both methods [rad]

The maximum error $\left(\max \left|y-y_{\text {est }}\right|\right)$ for both methods was close to $2.5 \cdot 10^{-2}$ rad but variance of the error in the first method was bigger $\left(5.48 \cdot 10^{-2}\right)$ than that in the second method $\left(4.15 \cdot 10^{-2}\right)$.

The second method is much simpler and gives very good speed estimation results for the BLDC motor. The estimated angle value in both cases is sufficiently precise. Sensorless drive for the BLDC motor can be important in small motors where the hall sensors enlarge the motor size. 


\section{Conclusions}

By selecting the $\mathbf{Q}$ and $\mathbf{R}$ matrices in a simulation way, the most favourable Kalman filter parameter can be determined to obtain a small error value in the steady state, but it requires a time-consuming simulation. Selecting the matrices by the second method is simpler and guarantees good estimation of speed and angle. It should also be remembered that the estimated result can be adjusted by changing the specific value of the covariance matrices and initial values of the filter. Filter tuning may also cause a change in overshoot and response time value. Therefore, one should consider whether the observer is fast enough and whether the overshoot does not exceed the assumed level. It should also be remembered that at the filter design stage, we are limited to determining the dynamics of the covariance matrix and it may turn out that the desired dynamics is not possible to achieve. A possible solution to this problem is to choose a different motor model, which can be extremely difficult.

\section{References}

Aishwarya, V. and Jayanand, B. (2016). Estimation and control of sensorless brushless DC motor drive using extended Kalman filter. In: IEEE International Conference on Circuit, Power and Computing Technologies (ICCPCT), India.

Auger, F., Hilairet, M., Guerrero, J. M., Monmasson, E., Orlowska-Kowalska, T. and Katsura, S. (2013). Industry Applications of the Kalman Filter: A Review. IEEE Transactions on Industrial Electronics, 60, pp. 5458-5471.

Dhaouadi, R., Mohan, N. and Norum, L. (1991). Design and Implementation of an Extended Kalman Filter for the State Estimation of a Permanent Magnet Synchronous Motor. IEEE Transactions on Power Electronics, 6(3), pp. 491-497.

Eissa, M. A., Ahmed, M. S., Darwish, R. R. and Bassiuny, A. M. (2015). Improved fuzzy Luenberger observerbased fault detection for BLDC motor. In: 2015 Tenth International Conference on Computer Engineering \& Systems (ICCES), Cairo, IEEE, pp. 167-174. doi: 10.1109/ICCES.2015.7393039.

El Mekki, A. and Ben Saad, K. (2016). A BLDC fault diagnosis approach based on a super-twisting sliding mode observer. In: 2016 4th International Conference on Control Engineering \& Information Technology (CEIT), Hammamet, IEEE, pp. 1-5. doi: 10.1109/CEIT.2016.7929039.

Jethwani, A., Aseri, D., Singh, T. S. and Jain, A. K. (2016). A simpler approach to the modelling of permanent magnet brushless DC machine in MATLAB environment. In: 6th IEEE International Conference on Power Systems (ICPS), India.

Lenine, D., Rami Reddy, B. and Vijay Kumar, S. (2007). Estimation of speed and rotor position of BLDC motor using extended Kalman filter. In: IET-UK International
Conference on Information and Communication Technology in Electrical Sciences (ICTES 2007), Chennai, Tamil Nadu, India, 20-22 December 2007, Dr. M.G.R. University, pp. 433-440.

Ryba, Ł., Voda A., Besancon G. (2014). An LQG/ LTR Approach Towards Piezoactuator Vibration Reduction With Observer-Based Hysteresis Compensation. IFAC Proceedings Volumes, 47(3), pp. 5623-5628.

Matlab Simulink. (2018). Mathworks: Permanent Magnet Synchronous Machine. Available at: https://www. mathworks.com [Accessed Jan. 2018].

Simon, D. (2006). Optimal State Estimation: Kalman, $H$ Infinity, and Nonlinear Approaches. USA: WileyInterscience. [ISBN: 978-0-471-70858-2].

Šlapák, V., Kyslan, K., Fedák, V. and Ďurovský, F. (2016). Finite Control Set Model Predictive Speed Control of a DC Motor. Mathematical Problems in Engineering, 2016, p. 10 (9571972). doi: 10.1155/2016/9571972.

Terzic, B. and Jadric, M. (2001). Design and Implementation of the Extended Kalman Filter for the Speed and Rotor Position Estimation of Brushless DC Motor. IEEE Transactions on Industrial Electronics, 48(6), pp. 1065-1073.

Vinida, K. and Chacko, M. (2016). An optimized H infinity strategy for robust control of sensorless BLDC propulsion motor in submarines for improved maneuverability. In: 2016 IEEE International Conference on Power Electronics, Drives and Energy Systems (PEDES), Trivandrum, IEE, pp. 1-6.

Zabalawi, S. A. and Nasiri, A. (2007). State space modeling and simulation of sensorless control of brushless DC motors using instantaneous rotor position tracking. In: IEEE Vehicle Power and Propulsion Conference (VPPC), Arlington, TX, USA. 